\title{
Editorial
}

\section{Odontología digital}

Juan Manuel Olarte-Sarmiento ${ }^{1 凶}$, Santiago Castro-Osorno ${ }^{1 凶}$

${ }^{1}$ Rehabilitador Oral. Fundador de Toothprint. Universidad CES, Medellin, Colombia.

Fecha correspondencia:

Recibido: noviembre de 2021.

Aprobado: diciembre de 2021.

Forma de citar:

Olarte-Sarmiento JM, Castro-

Osorno S. Odontología digital.

CES Odont 2021; 34(2): 1-2.

https://doi.org/10.21615/

cesodon.6539

\section{Open access}

(C) Derecho de autor

Licencia creative commons

Ética de publicaciones

Revisión por pares

Gestión por Open Journal System

DOI: $10.21615 /$ cesodon. 6539

ISSNe 2215-9185

ISSN 0120-971X

Publica con nosotros
Cuando nos mencionan el término odontología digital, pensamos siempre en equipos vanguardistas de última tecnología. Aunque si es cierto que involucra todas estas herramientas, como su nombre lo dice, son herramientas. Pero la odontología es la misma, nuestras bases biológicas, fisiológicas e histológicas son las mismas. Siempre han sido las mismas, y lo seguirán siendo. La odontología digital está presente desde hace 35 años y se está abriendo camino en las prácticas de cada uno de nosotros. Desde el punto de vista profesional su mayor ventaja es la integración de múltiples archivos. Archivos 2D como las fotografías, radiografías, archivos 3D como escaneos intraorales, escaneos extraorales, escaneos faciales y escaneos tomograficos. Actualmente estamos interactuando con archivos 4D, son archivos dinámicos que nos permiten capturar movimientos específicos de los pacientes. Esta integración de manera analógica no era posible realizarla, la manipulación de esta información solo servía de ayuda diagnostica. Pero es hoy cuando esta integración de archivos nos permite diagnosticar mucho mejor, si estamos diagnosticando mejor, nuestros planes de tratamiento serán mejores y más predecibles. Cuando trasladamos cada situación específica, cada movimiento, cada condición maxilo-mandibular, ósea, periodontal y dental creamos el concepto: "PACIENTE DIGITAL". Aquí está toda la información que recolectamos durante la fase diagnóstica, ahora de manera sincrónica o asincrónica comenzamos la interacción alrededor de estas herramientas digitales con todos los miembros de nuestro equipo interdisciplinario de trabajo. Todas las especialidades se van a nutrir alrededor de estas herramientas y con la creación del 
paciente digital, migraremos a el concepto de "PACIENTE VIRTUAL" donde crearemos tantos planes de tratamiento queramos, justo en la pantalla del computador y antes de comenzar los procedimientos clínicos con el paciente. En la manipulación de los archivos 2D, 3D y 4D podemos empezar a interactuar y comunicarnos mejor con los Dres involucrados en las fases de tratamiento y más aun con nuestros pacientes creando un vínculo más sólido en la decisión y aceptación de los planes de tratamiento. La falta de entendimiento de los planes de tratamiento por parte de los pacientes era un común denominador. Con la creación del "PACIENTE VIRTUAL", podemos aterrizar toda la información que los pacientes investigan y todo nuestro conocimiento profesional para presentar el diagnóstico, las simulaciones de los tratamientos de una manera gráfica, didáctica y práctica dejando siempre al paciente en el centro de la ecuación. Dentro del paciente virtual se establecen tiempos de trabajo y el presupuesto del tratamiento. Una vez se dé la aceptación del plan de tratamiento, entran softwares robustos enfocados en cada especialidad que permitirán el desarrollo del procedimiento dentro del paciente virtual para luego con impresión 3D y máquinas de control numérico como las fresadoras a trasladar todas las planificaciones, planes de tratamiento y diseños que teníamos en nuestras pantallas a la vida real y crear el concepto de "PACIENTE REAL". En esta fase tenemos interacción física para los procedimientos clínicos gracias a los diferentes tipos de manufactura empleadas, trasladamos las simulaciones en objetos físicos con toda la información de las planificaciones. Los pacientes verán el reflejo de las planificaciones como ventaja ya que el número de citas para los planes de tratamiento serán menores y los tiempos clínicos de los procedimientos se reducirán por la consistencia y precisión ya que las maquinas son excelentes produciendo y nosotros los humanos guiándolas. 